\title{
SM-CO BASED MAGNETIC SYSTEM FOR 10 MEV TECHNOLOGICAL ELECTRON ACCELERATOR LU-10M
}

\author{
V.A. Bovda, A.M. Bovda*, I.S. Guk, S.G. Kononenko, V.N. Lyashchenko, A.O. Mytsykov, \\ L.V. Onischenko \\ NSC "Kharkiv Institute of Physics and Technology" NASU \\ Kharkiv, 61108, st. Akamemicheskaya 1, Ukraine \\ *E-mail:bovda@kipt.kharkov.ua,https://orcid.org/0000-0002-1684-1475 \\ Received 31 May 2018; revised 3 August 2018; accepted 17 September 2018
}

Rare-earth permanent magnets are widely used in the accelerators of charged particles. However, the magnetic performance under irradiation remains a key issue for the most high energy applications such as accelerators with the energy up to $10 \mathrm{MeV}$. The aim of the work was to assess radiation and magnetic stability of Sm-Co and Nd-Fe-B permanent magnets under the direct electron irradiation with the energy of $10 \mathrm{MeV}$ and bremsstrahlung. Sm-Co and Nd-Fe-B permanent magnets were produced by powder metallurgy method including PLP for the latter. The absorbed dose imposed by electron beam was 16 Grad (the total flux of electron per $1 \mathrm{~cm}^{2}$ was $1.4 \times 10^{17}$ ) and $160 \mathrm{Grad}$. The radiation activity of both Nd-Fe-B and Sm-Co magnets was within the acceptable limits after the irradiation. This makes rare-earth magnetic materials suitable for such applications. In order to avoid overheating during electron irradiation, magnets were cooled with the water $\left(\mathrm{T}=38^{\circ} \mathrm{C}\right)$. In order to estimate the changes in magnetic flux, the integral of the 3D interpolation normal component of magnetic flux was used. Calculated $\mathrm{S}$ parameter measured in arbitrary units was chosen as integrated z-component of magnetic flux. It was shown that magnetic flux of Nd-Fe-B magnets became 0.92 and 0.717 of initial values for $16 \mathrm{Grad}$ and $160 \mathrm{Grad}$ correspondingly, but the magnetic flux of Sm-Co magnets had no change to the same absorbed doses. Thus, Sm-Co magnets were chosen for simulating and designing magnetic system for electron beam analysis of a technological accelerator with energy up to $10 \mathrm{MeV}$. The distance between the poles of the magnet was $25.25 \mathrm{~mm}$. The highest magnetic field inside the magnetic system was $0.3110 \mathrm{~T}$. The effective distance was $33.53 \mathrm{~mm}$. The measured parameters of the magnetic system based on Sm-Co magnets agreed with the simulation experiment. Magnetic system can also be used to adjust the accelerator in the energy range up to $10 \mathrm{MeV}$.

KEY WORDS: Nd-Fe-B, $\mathrm{Sm}_{2} \mathrm{Co}_{17}$, permanent magnet, electron accelerator, magnetic field

\section{АНАЛІЗУЮЧИЙ МАГНІТ ДЛЯ ТЕХНОЛОГІЧНОГО ПРИСКОРЮВАЧА ЕЛЕКТРОНІВ ЛУ-10М В.О. Бовда, О.М. Бовда, І.С. Гук, С.Г. Кононенко, В.М. Лященко, А.О. Мициков, Л.В. Оніщенко Наиіональний Науковий Центр «Харківський фізико-технічний інститут» 61108, Харків, Академічна, 1}

Основною проблемою при використанні магнітів з рідкісноземельних матеріалів в прискорювальних технологіях $є$ зміна магнітних властивостей матеріалу під дією випромінювання. Особливо актуальна ця задача для потужних технологічних прискорювачів електронів 3 енергією до $10 \mathrm{MeB}$. Для вибору найбільш стійкого матеріалу були проведені дослідження радіаційної стійкості зразків магнітів $3 \mathrm{Sm}_{2} \mathrm{Co}_{17}$ i Nd-Fe-B сплаву. Постійні магніти типу $\mathrm{Sm}_{2} \mathrm{Co}_{17}$ i Nd-Fe-B були виготовлені методом порошкової металургії з використанням PLP процесу при виробництві останніх. Магнітні зразки піддавалися прямому впливові електронного пучка з енергією $10 \mathrm{MeB}$ і впливові гальмівного випромінювання цього пучка. Поглинена доза від електронів для зразків складала 16 Град (загальний потік електронів, що потрапив на $1 \mathrm{~cm}^{2}$ зразка, дорівнює $\left.1,4 \times 10^{17}\right)$ i 160 Град. Активність зразків після опромінення змінювалась у межах допустимих меж. Цей факт значно спрощує використання рідкісноземельних магнітних матеріалів у готових виробах прискорювачів. Активність зразків після опромінення змінилася в межах припустимих норм. Цей факт істотно спрощує використання магнітних матеріалів у готових виробах. У процесі опромінення магніти охолоджувалися водою з температурою $38^{\circ} \mathrm{C}$ для запобіганню перегріву. Для оцінки зміни магнітного поля, що утворюється навколо зразка, використано інтеграл інтерпольованої нормалі до поверхні зразка складової магнітного поля $\mathrm{S}$ по області інтерполюваннях даних в умовних одиницях. Для зразків з $\mathrm{Nd}-\mathrm{Fe}-\mathrm{B}$ сплаву магнітний потік навколо зразка зменшився і становив 0,92 і 0,717 початкового значення для зазначених доз опромінення. Магнітне поле навколо зразків $3 \mathrm{Sm}_{2} \mathrm{Co}_{17}$ сплаву не змінилося в межах точності вимірів для тих же доз опромінення. На основі зразків з $\mathrm{Sm}_{2} \mathrm{Co} 17$ сплаву розмірами $30 \mathrm{x} 24 \mathrm{x} 12$ мм було проведене моделювання і конструювання магніту для аналізу пучка електронів технологічного прискорювача на енергію до $10 \mathrm{MeB}$. Найбільша величина поля в медіанній площині магніту становила 0,3110 Тл. Відстань між полюсами магніту дорівнює 25,25 мм. Ефективна довжина магніту - 33,53 мм. Виміряні параметри поля магніту задовольняють закладеним при розробці величинам. Магніт може бути використано для налагодження прискорювача в діапазоні енергій до $10 \mathrm{MeB}$.

КЛЮЧОВІ СЛОВА: Nd-Fe-B, $\mathrm{Sm}_{2} \mathrm{Co}_{17}$, постійні магніти, прискорювач електронів, поле магніту

\section{АНАЛИЗИРУЮЩИЙ МАГНИТ ДЛЯ ТЕХНОЛОГИЧЕСКОГО УСКОРИТЕЛЯ ЭЛЕКТРОНОВ ЛУ-10М В.А. Бовда, А.М. Бовда, И.С. Гук, С.Г. Кононенко, В.Н. Лященко, А.О. Мыцыков, Л.В. Онищенко Наииональный Научный Центр «Харьковский физико-технический институт» 61108, Харьков, Академическая, 1}

Основной проблемой при использовании магнитов из редкоземельных материалов в ускорительных технологиях является изменение магнитных свойств материала под действием излучения. Особенно актуальна эта задача для мощных технологических ускорителей электронов с энергией до 10 МэВ. Для выбора наиболее стойкого материала были проведены 
исследования радиационной стойкости образцов магнитов из $\mathrm{Sm}_{2} \mathrm{Co}_{17}$ и $\mathrm{Nd}-\mathrm{Fe}-\mathrm{B}$ сплава. Постоянные магниты типа $\mathrm{Sm}_{2} \mathrm{Co}_{17}$ и Nd-Fe-B были изгтовлены методом порошковой технологи с использованием PLP процесса в производстве последних. Магнитные образцы подвергались прямому воздействию электронного пучка с энергией 10 МэВ и воздействию тормозного излучения этого пучка. Поглощённая доза от электронов для образцов составляла 16 Град (общий поток электронов, попавший на $1 \mathrm{~cm}^{2}$ образца, был равен $1,4 \times 10^{17}$ ) и 160 Град. Активность образцов после облучения изменилась в пределах допустимых норм. Этот факт существенно упрощает использование редкоземельных магнитных материалов в готовых изделиях ускорителей. В процессе облучения магниты охлаждались водой с температурой $38^{\circ} \mathrm{C}$ для избежания перегрева.

Для оценки изменения величины поля, создаваемого вокруг образца, использовался интеграл интерполированной нормальной к поверхности образца составляющей магнитного поля $\mathrm{S}$ по области интерполяции данных в относительных единицах. Для образцов из Nd-Fe-B сплава магнитный поток вокруг образца уменьшился и составил 0,92 и 0,717 от начального значения для указанных доз облучения. Магнитное поле вокруг образцов из $\mathrm{Sm}_{2} \mathrm{Co}_{17}$ сплава не изменилось в пределах точности измерений для тех же доз. На основе образцов из $\mathrm{Sm}_{2} \mathrm{Co}_{17}$ сплава размерами $30 \times 24 \mathrm{x} 12$ мм было проведено моделирование и конструирование магнита для анализа пучка электронов технологического ускорителя на энергию до 10 МэВ. Наибольшее значение поля в медианной плоскости магнита равно 0,3110 Тл. Расстояние между полюсами магнита равно 25,25 мм. Эффективная длина магнита - 33,53 мм. Измеренные параметры поля магнита удовлетворяют заданным при разработке величинам. Магнит также может быть использован для настройки ускорителя в диапазоне энергий до 10 МэВ.

КЛЮЧЕВЫЕ СЛОВА: Nd-Fe-B, $\mathrm{Sm}_{2} \mathrm{Co}_{17}$, постоянные магниты, ускоритель электронов, поле магнита

Использование магнитов на основе сплавов редкоземельных элементов позволяет создавать дипольные магниты для широкого класса ускорителей. Это медицинские и технологические линейные ускорители, микротроны, линейные коллайдеры [1-5]. Создание каждого такого устройства связано с необходимостью учёта параметров ускорителей и особенностей их эксплуатации. Линейный ускоритель ЛУ-10 [6] в ННЦ ХФТИ много лет используется для технологической радиационной обработки материалов и изделий электронным пучком с энергией около 10 МэВ. Сейчас проводится модернизация всех систем ускорителя. Предполагаемые параметры пучка этого ускорителя [7] после модернизации систем представлены ниже.

Основные параметры пучка ускорителя ЛУ-10М:

Ток пучка, A - 0,389

Энергия в максимуме, МэВ - 9,44

Энергетический спектр (99\% част.), \% - 51

Энергетический спектр на половине высоты, \% - 0,48

Диаметр (99 \% част.), мм - 6,8

Среднеквадратичный радиус, мм - 1,5

Среднеквадратичный нормализованный эмиттанс, мм мрад - 13

На основе этих параметров были выбраны схемы каналов вывода пучка на ускорителе [8]. Эти параметры были использованы при выборе основных требований к магниту для измерения энергии пучка ускорителя [9]. Согласно выводам этой работы, конструкция магнита должна обеспечивать получение поля величиной около 0,3 Тл в зазоре 2,5 см.

Целью этой работы являлось исследование радиационной стойкости и выбор магнитного материала для использования в магните. На основе полученных результатов разработана конструкция магнита и проведены исследования характеристик поля магнита.

\section{ВЫБОР МАГНИТНОГО МАТЕРИАЛА}

Одной из главных особенностей использования постоянных магнитов на технологических ускорителях электронов является наличие сильного радиационного фона. Поэтому при выборе магнитного материала необходимо руководствоваться требованием сохранения магнитных свойств при больших дозах облучения электронным пучком и тормозным излучением этого пучка. Имеющиеся в литературе сведения о радиационной стойкости материалов из $\mathrm{Sm}-\mathrm{Co}$ и $\mathrm{Nd}-\mathrm{Fe}-\mathrm{B}$ сплава [10-23] под действием электронов с энергиями от 8 ГэВ до 10 МэВ и гамма квантов не позволяют достаточно однозначно выбрать материал для магнита. В связи с этим были проведены экспериментальные исследования изменения магнитного поля образцов магнитов из этих сплавов при прямом облучении электронным пучком с энергией 10 МэВ и тормозным излучением этого пучка $[24,25]$.

Магниты изготавливались методами порошковой металлургии [26]. В серии измерений были использованы образцы магнитов, предварительно намагниченных в импульсном магнитном поле 3,5 Тл. Геометрические размеры магнитов - 30х24x12 мм. Поверхность образцов из Nd-Fe-B для предотвращения коррозии была покрыта слоем никеля толщиной 0,02 мм.

Образцы облучались электронным пучком линейного технологического ускорителя КУТ-1 [27] с энергией 10 МэВ. Ось ускорителя располагалась вертикально, пучок выводится сверху вниз. Развернутый магнитом с изменяемым полем пучок электронов выводился из ускорителя в воздух через титановую фольгу. Образцы ориентировались таким образом, что в процессе облучения электроны падали на южный полюс магнита размером 30х24 мм. Плотность потока электронов в пределах размеров образца не изменялась больше, чем на $10 \%$. В процессе облучения образцы охлаждались водой с температурой не более $40^{\circ} \mathrm{C}$. 
В измерениях использовались по 4 образца магнитов, изготовленных из Sm-Co и Nd-Fe-B сплава.

Образцы №1, №2 облучались непрерывно в течение 20 часов. Электронный пучок падал прямо на поверхность образца №1. Поглощённая доза от электронов для образца №1 составляла 16 Град (общий поток электронов, попавший на $1 \mathrm{~cm}^{2}$ образца, был равен $1,4 \times 10^{17}$ ). Образец №2 облучался одновременно с образцом №1, но располагался вне электронного пучка на расстоянии 40 мм от него. Образец №3 облучался сеансами длительностью по 20 часов с перерывами 24 часа. Суммарная поглощённая доза для этого образца составила 160 Град. Образец №4 не подвергался воздействию облучению и служил опорным для магнитных и радиационных измерений.

Измерения для образцов из $\mathrm{Nd}-\mathrm{Fe}-\mathrm{B}$ сплава указывают на появление в результате реакции ${ }^{148} \mathrm{Nd}(\gamma, \mathrm{n})^{147} \mathrm{Nd}$, порог которой равен 7,3 МэВ, небольшого количества нестабильного изотопа ${ }^{147} \mathrm{Nd}$, с периодом полураспада 10,98 дней. Для образцов из $\mathrm{Sm}-$ Со сплава спектр характерен для изотопа ${ }^{153} \mathrm{Sm}$, образованного в результате реакции ${ }^{154} \mathrm{Sm}(\gamma, \mathrm{n}){ }^{153} \mathrm{Sm}$, идущей под действием тормозного излучения электронов. Время жизни этого изотопа равно 46,284 часа. После облучения активность образцов магнитов изменяется несущественно и не ограничивает возможности нормальной работы с изделиями, в которых эти магниты используются.

\section{ИЗМЕРЕНИЕ МАГНИТНОГО ПОЛЯ ВОКРУГ ОБРАЗЦОВ}

Для измерений распределения магнитного поля вокруг образцов использовалась линейка из семи датчиков Холла, закреплённых в массивной медной матрице для выравнивания температуры датчиков. Расстояние между датчиками в матрице изменялось от 6,48 до 7,034 мм. Измерялась нормальная по отношению к поверхности образца составляющая поля. Относительная погрешность измерений поля датчиками была не хуже $0,01^{\circ} \%$.

Образцы передвигались параллельно поверхности матрицы на расстоянии, равном 3,05 мм. Расстояние между точками измерения вдоль движения магнита изменялось от 3 до 5 мм. Величина перемещения измерялась с точностью 1 микрон. Начальная точка измерения для всех образцов фиксировалась с помощью специального упора. Поле для всех образцов измерялось как со стороны южного, так и со стороны северного полюса магнита (более подробная информация о методике измерений содержится в работе [24]).

По измеренным точкам была проведена трёхмерная квадратичная интерполяция полученных данных между точками измерения. Размер области, в которой проводилась интерполяция данных, задавался расстоянием между крайними датчиками матрицы, а вдоль движения образца - областью сканирования, точно задаваемой системой определения координат образца. На рис. 1 представлены результаты такой интерполяции для измерений, выполненных для образца № 1 из Nd-Fe-B сплава до облучения.

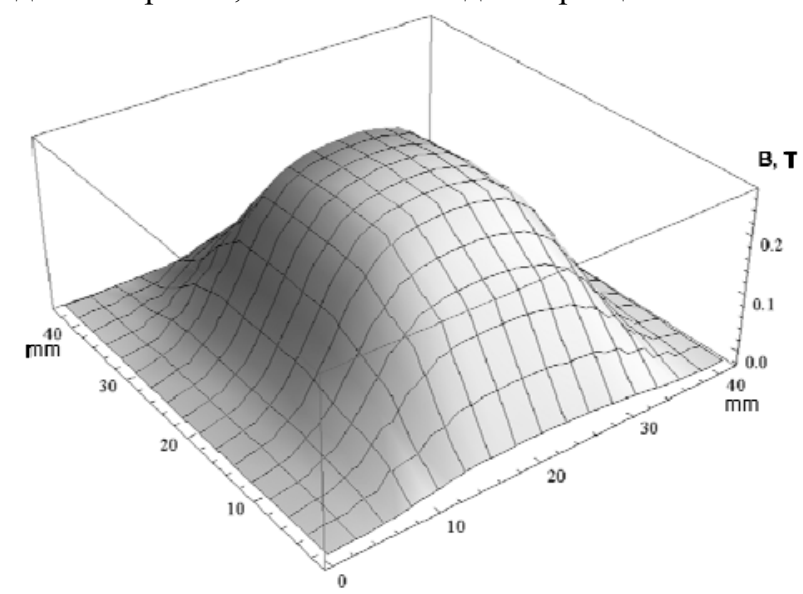

Рис. 1. Распределение поля до облучения образца №1.

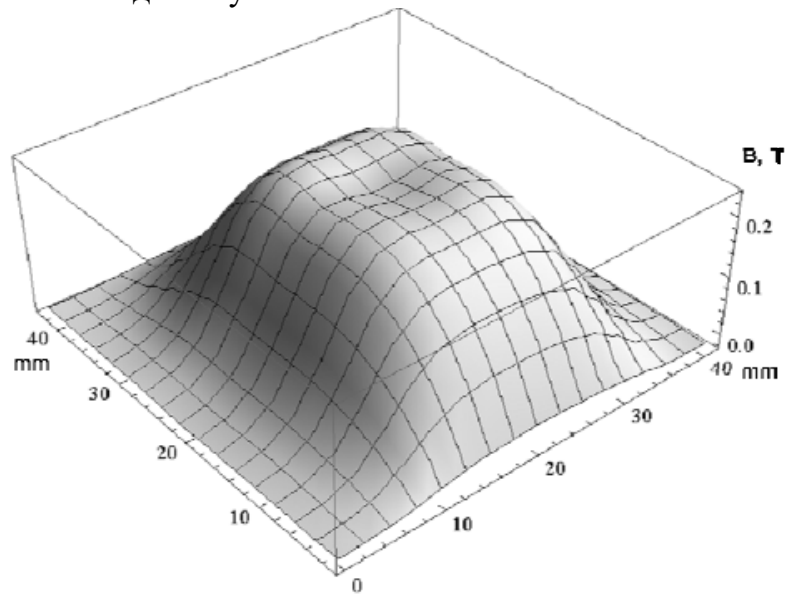

Рис. 2. Распределение поля после облучения образца №1. Поглощённая доза составляла 16 Град.

Для оценки изменения величины поля, создаваемого вокруг образца, использовался интеграл интерполированной нормальной к поверхности образца составляющей магнитного поля $\mathrm{S}$ по области интерполяции данных в относительных единицах. Проведенные исследования точности повторения этой меры для одного и того же образца, связанной с привязкой к границам образца измерительной системы, дают повторяемость на уровне $0,5 \%$.

Величина этого интеграла для поля, измеренного со стороны северного полюса использованных в исследовании образцов $\mathrm{Nd}-\mathrm{Fe}-\mathrm{B}$ сплава до начала облучения, приведена ниже.

$\mathrm{S}_{1}=175,763 ; \mathrm{S}_{2}=179,556 ; \mathrm{S}_{3}=176,357 ; \mathrm{S}_{4}=175,452$

Эти величины совпадают с данными, полученными для аналогичных измерений поля со стороны южного полюса этих образцов в пределах точности измерений.

Данные по распределению поля со стороны северного полюса, полученные после облучения образца №1 электронным пучком, представлены на рис. 2. Величина $\mathrm{S}_{1}$ при этом изменилась до значения 162,356.

Распределение поля со стороны южного полюса этого образца после облучения представлено на рис. 3. 
Величина $\mathrm{S}_{1}$ для этих данных равна -160,2.

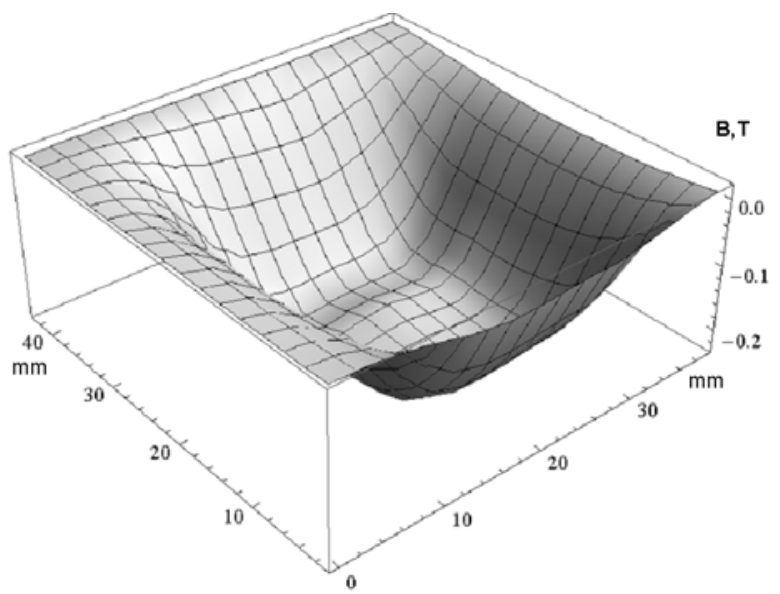

Рис. 3. Распределение поля после облучения образца №1 со стороны южного полюса. Поглощённая доза составляла 16 Град.

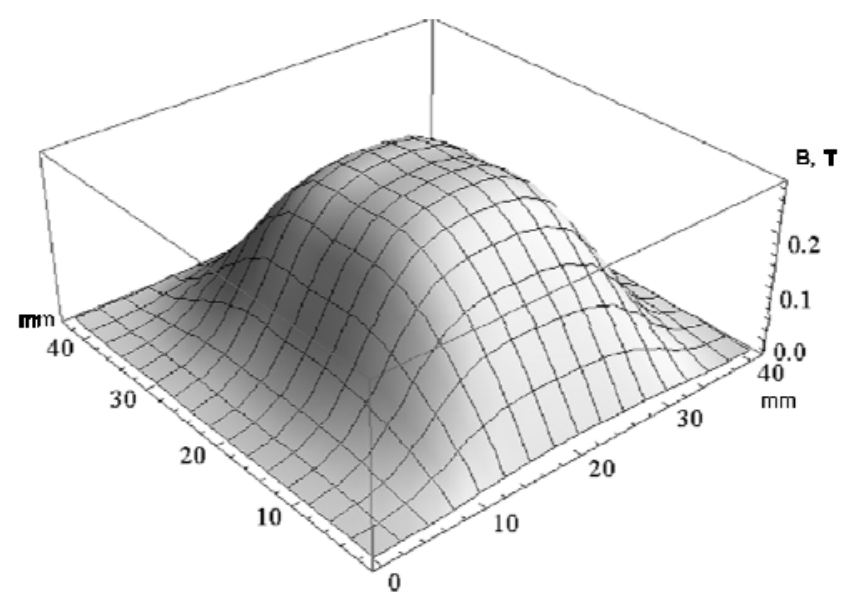

Рис. 4. Распределение поля со стороны северного полюса после облучения образца №2.

Как можно видеть из рис.2 и рис.3, после облучения как распределение поля со стороны северного и южного полюсов магнита, так и величина интеграла поля с обеих сторон в пределах точности измерений совпадают.

Распределение поля после облучения образца №2 тормозным излучением (рис.4) не отличается от первоначального, а величина интеграла $\mathrm{S}_{2}=178,526$ также практически не изменилась.

Наибольшие изменения распределения поля вокруг магнита наблюдались после облучения образца №3 (рис.5), величина $S_{3}$ для него уменьшилась до значения 126,556. Однако при этом необходимо заметить, что интеграл $\mathrm{S}$ поля после облучения не изменился пропорционально изменению величины поглощенной дозы.

Для контрольного образца измерения распределения поля после окончания цикла измерений на облучаемых образцах показали совпадение с распределениями, полученными до облучения.

Аналогичные измерения были проведены для образцов из Sm-Со сплава. Величина интеграла поля с южного полюса для образца №1 равна -151,94; для №2 -149,007; для №3 -152,326; №4 -152,519. Величины интеграла поля с северного полюса для всех этих образцов в пределах точности измерений совпадают с приведенными выше для южного полюса. рис. 6.

Распределение поля со стороны северного полюса вокруг образца №1 до облучения представлено на

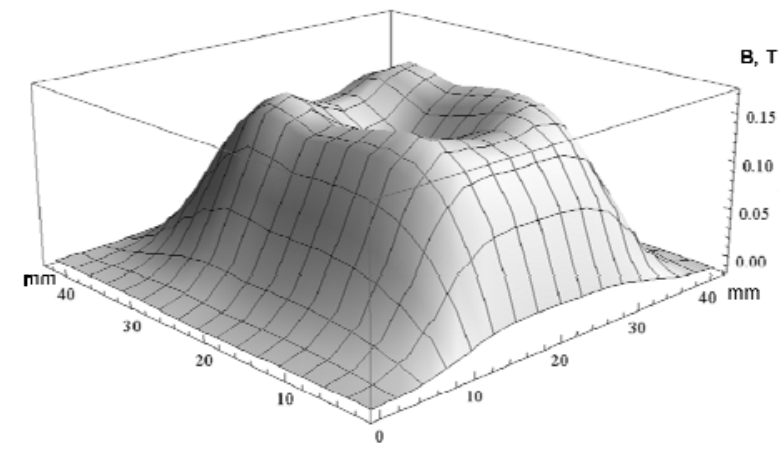

Рис. 5. Распределение поля магнита №3 после облучения. Поглощённая доза составляла 160 Град.

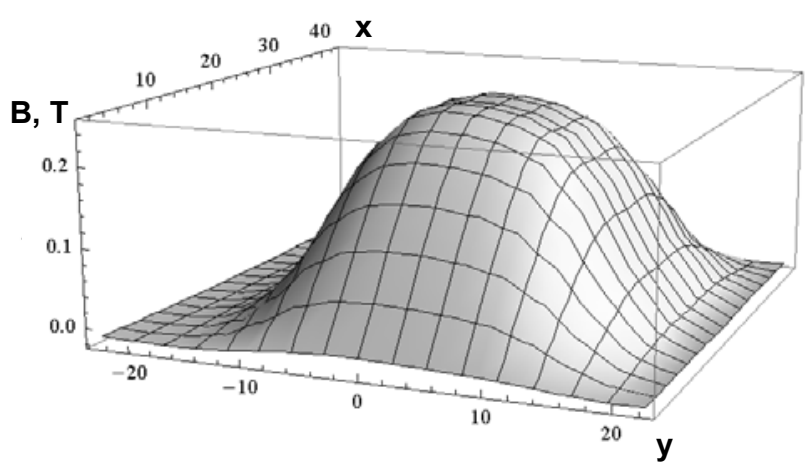

Рис. 6. Распределение поля магнита №1 до облучения. Северный полюс.

Распределение поля со стороны южного полюса вокруг образца №1 до облучения представлено на рис. 7.

Распределение поля со стороны южного полюса вокруг образца №1 после облучения представлено на рис.8. Оно совпадает с распределением поля этого образца до облучения.

Доза облучения для образца №3 в 160 Град не изменила первоначального распределения поля вокруг этого магнита. Интегралы поля после облучения равны для образца №2 - 148,397 и -149,727, для образца №3 149,714 и -150,065 для северного и южного полюсов соответственно.

Распределения поля вокруг образцов №2 и №3 после облучения симметричны относительно осей образцов и практически совпадают с распределениями до облучения.

Интеграл для магнита №4 после окончания измерений равен 152,496 и -155,135.

Таким образом, проведенные исследования показали преимущество использования в магните образцов из 
$\mathrm{Sm}-\mathrm{Co} \mathrm{сплава} \mathrm{по} \mathrm{сравнению} \mathrm{с} \mathrm{образцами} \mathrm{из} \mathrm{Nd-Fe-B} \mathrm{сплава,} \mathrm{поскольку} \mathrm{их} \mathrm{магнитные} \mathrm{свойства} \mathrm{не} \mathrm{меняются} \mathrm{под}$ воздействием прямого облучения электронным пучком и тормозным излучением этого пучка.

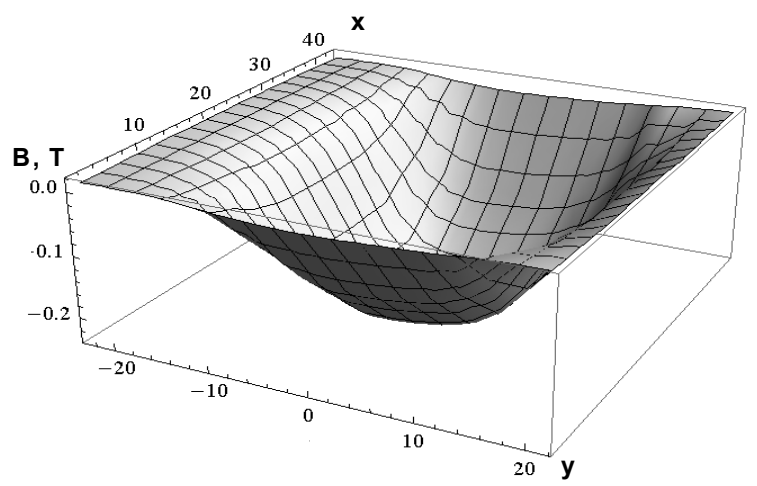

Рис. 7. Распределение поля магнита №1 до облучения.

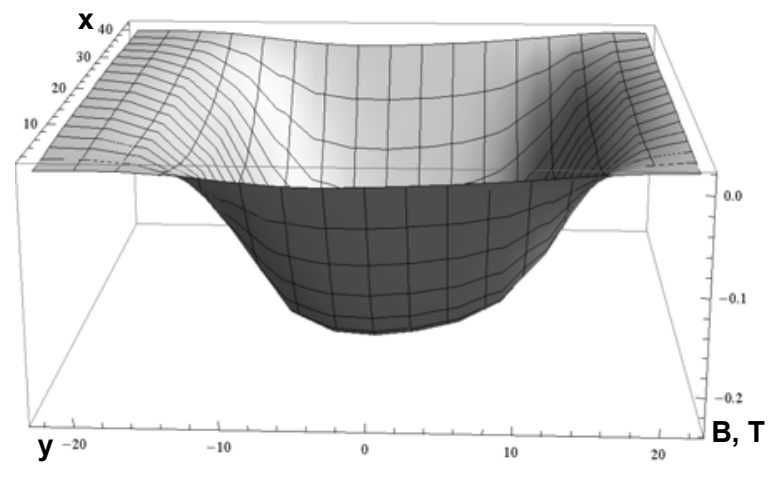

Рис. 8. Распределение поля магнита №1 после облучения. Поглощённая доза составляла 16 Град.

Кроме того, магниты из $\mathrm{Sm}_{2} \mathrm{Co}_{17}$ сплава имеют лучшие характеристики в тех случаях, когда в условиях, приближенных к критическим, изделие должно оставаться стабильным. Такие условия могут часто возникать при использовании магнитов на технологических ускорителях электронов. В случае работы при высоких температурах и в агрессивных условиях, изделия из $\mathrm{Sm}_{2} \mathrm{Co}_{17}$ сплава имеют определённые преимущества перед устройствами, изготовленными из $\mathrm{Nd}-\mathrm{Fe}-\mathrm{B}$ сплава. Температура Кюри $\mathrm{Sm}_{2} \mathrm{Co}_{17}$ сплава равна $825{ }^{\circ} \mathrm{C}$. Максимальная рабочая температура самариевых магнитов достигает 350 градусов Цельсия. Температура Кюри для магнитов из сплава Nd-Fe-B равна $312{ }^{\circ} \mathrm{C}$, поэтому в условиях реальных экспериментов рабочие температуры для этого сплава не превышают $180^{\circ} \mathrm{C}$.

Температурная стабильность магнитов изготовленных из Nd-Fe-B сплава меньше, чем у магнитов, изготовленных из $\mathrm{Sm}-$ Со сплава - их температурный коэффициент магнитной индукции изменяется от 0,07 до $0,13\left(\% /{ }^{0} \mathrm{C}\right)$ в противовес $-0,035\left(\% /{ }^{0} \mathrm{C}\right)$ у $\mathrm{Sm}-\mathrm{Co}$.

Кроме того, магниты из самариевого сплава меньше подвержены коррозии, чем неодимовые магниты, и обычно не требуют покрытия.

Полученные выше результаты позволили провести окончательный выбор на использование Sm-Co сплава в разработке магнита для ускорителя.

\section{МАГНИТ ДЛЯ УСКОРИТЕЛЯ ЛУ-10М}

Образцы постоянных магнитов, изготовленных из $\mathrm{Sm}$-Со сплава, размером 30х24x12 мм были использованы для оценки величины поля, которое может быть получено в дипольном магните. Магниты располагались в пространстве на расстоянии 25 мм. Максимальное значение поля, измеренное в медианной плоскости такого магнита, было близким к величине 0,3 Тл. Эта величина поля была использована при моделировании движения пучка на выходе ускорителя. Результаты моделирования позволили сформулировать окончательные требования к конструкции магнита.

На рис. 9 представлен образец магнита, конструкция которого была разработана и изготовлена на основе проведенного моделировния.

Внешние размеры магнитопровода - 90х73х24 мм. Он изготовлен из магнитомягкой стали Ст3. Точность изготовления и привязки элементов магнита -5 микрон. Магнитные образцы из Sm-Co сплава крепятся к балкам при помощи алюминиевых зажимов. Верхняя балка магнита съёмная. Это позволяет легко монтировать и демонтировать магнит на выходном фланце ускорителя при процедуре измерения или подстройки энергии ускорителя. Конструкция магнита позволяет использовать его для развёртки пучка как в горизонтальной плоскости, так и в вертикальной. Было проведено измерение распределения поля в магните с помощью описанной в предыдущем разделе работы методики. На рис. 10 представлены результаты измерений.

Распределения поля магнита, полученное в результате интерполирования экспериментальных данных, представлено на рис. 11. Это распределение поля магнита близко к распределению поля, полученному в результате предварительного выбора параметров магнита и моделирования распределения поля в нём [9].

Максимальное значение поля в магните равно 0,3110 Тл. Эффективная длина магнита вдоль центральной траектории пучка - 33,533 мм. Угол поворота пучка электронов с энергией 10 МэВ при движении через этот магнит, согласно моделированию движения, равен 0,31 рад.

Было исследовано изменение магнитного поля в магните при размыкании и замыкании магнитного потока в магнитопроводе при удалении и восстановлении положения верхней балки магнита. На рис. 12 представлены результаты измерений поля в семи точках датчиками Холла. Датчики располагались в центре магнита. В течение 1000 секунд было проведено 10 циклов размыкания - замыкания поля с помощью верхней балки магнита. 


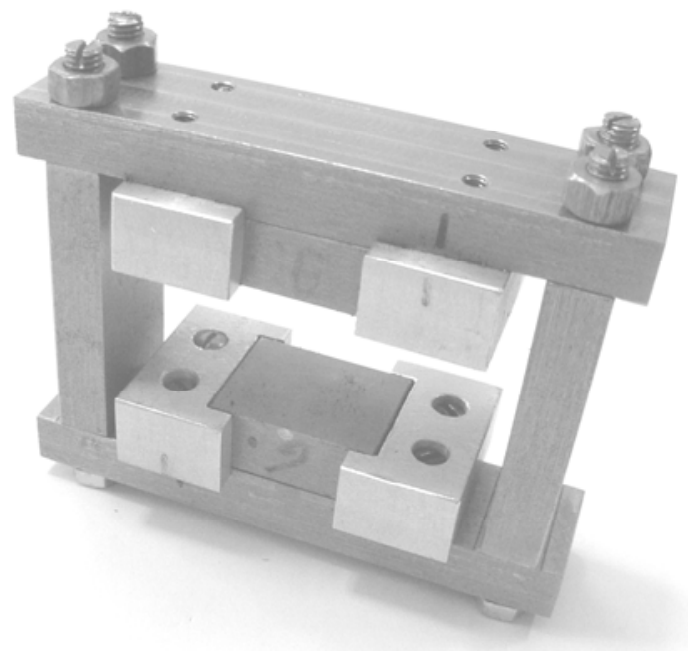

Рис. 9. Магнит в собранном виде.

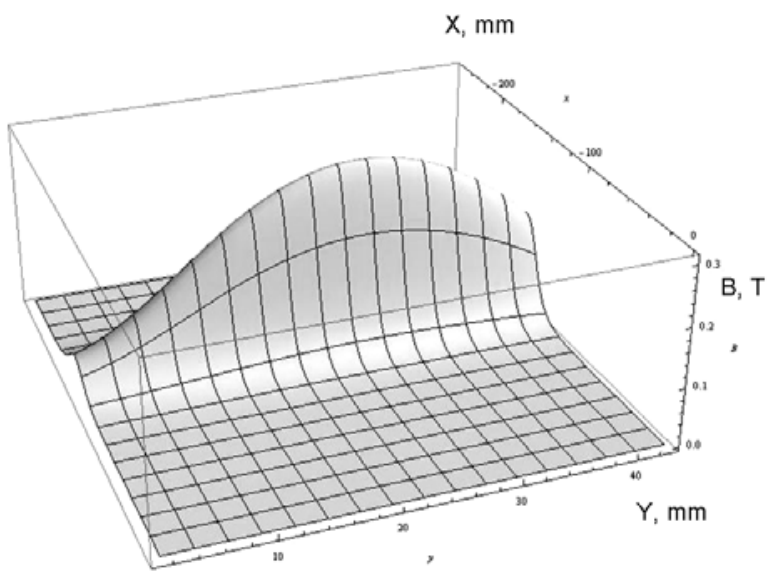

Рис. 11. Распределение поля в магните.

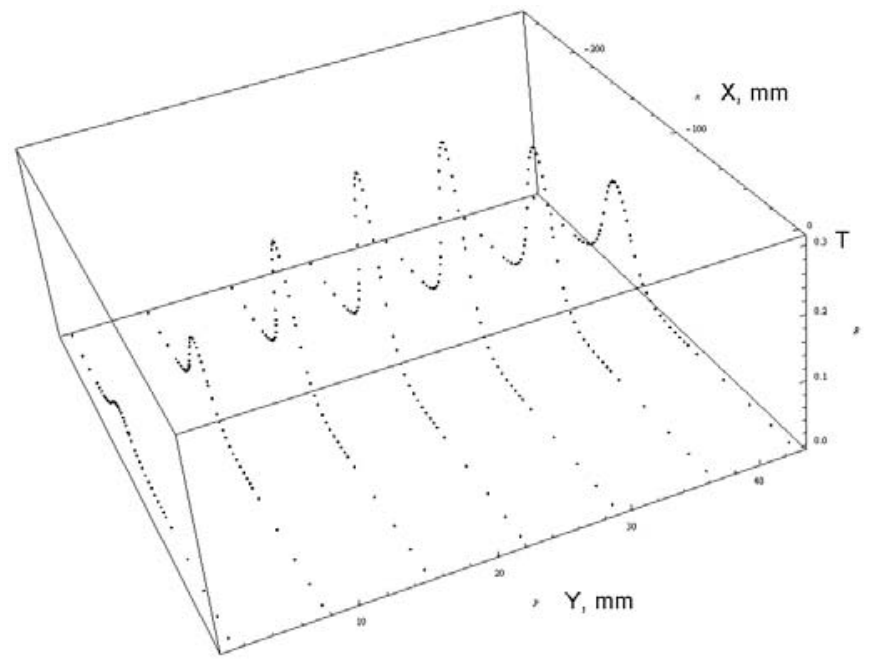

Рис. 10. Результаты измерений поля датчиками Холла.

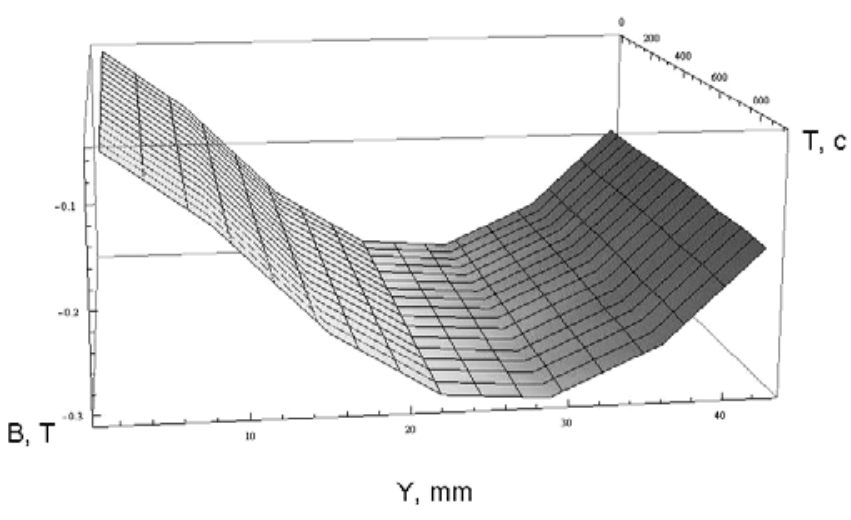

Рис. 12. Распределение поля в магните после цикла размыкание - замыкание поля.

Относительное изменение величины поля в точках измерения представлено на рис. 13. Цифры на рисунке обозначают номера датчиков. Для датчиков, расположенных вблизи центра магнита (номера датчиков 3, 4 и 5), максимальное изменение поля после замыкания поля не превышало 0,5 \%.

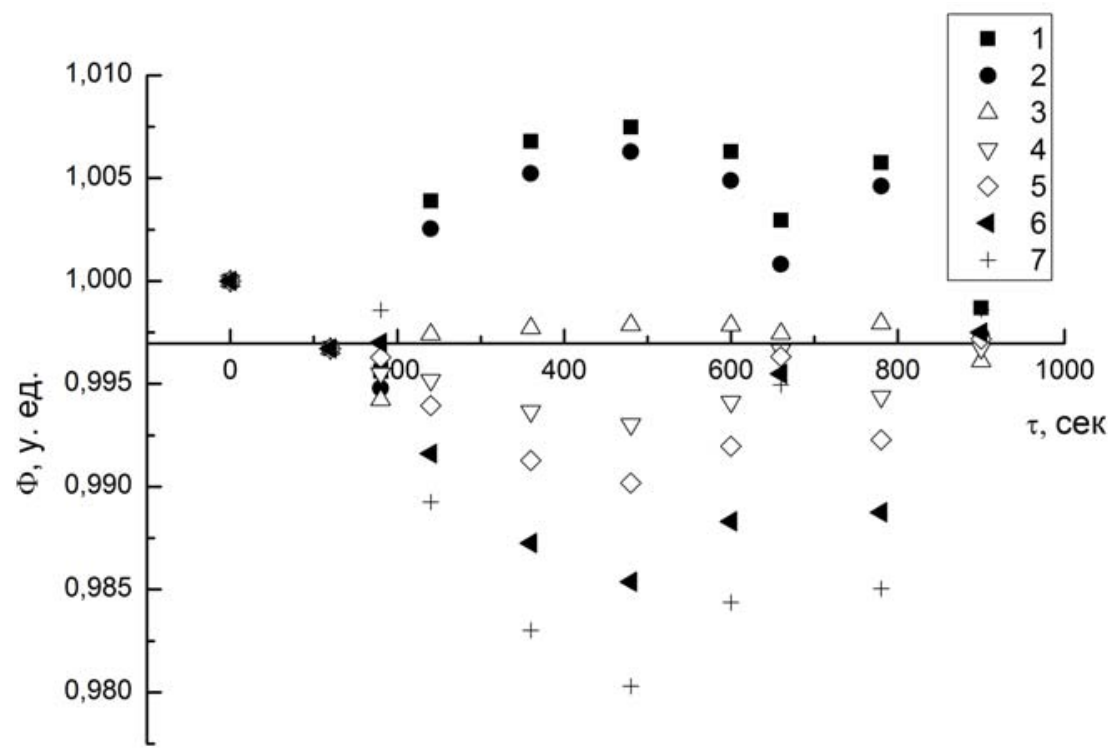

Рис. 13. Относительное изменение величины поля в местах расположения датчиков Холла. 


\section{РЕЗУЛЬТАТЫ}

Экспериментально обоснован выбор магнитного сплава для разработки магнита, позволяющего сохранять магнитные свойства в условиях высоких уровней облучения электронами и тормозным излучением электронного пучка. Разработан и изготовлен с использованием магнитов из Sm-Со сплава дипольный магнит для измерения энергии технологического ускорителя до 10 МэВ. Измеренные параметры поля магнита удовлетворяют заданным при разработке величинам. Магнит также может быть использован для настройки ускорителя в диапазоне энергий до 10 МэВ.

\section{СПИСОК ЛИТЕРАТУРЫ}

1. Shvedunov V. I., et al., A 70 MeV Race-Track Microtron // Nucl. Instr. Meth. - 2005. - Vol. 550. - No. 1-2. - P. $39-53$.

2. David J. McLaughlin, Kenneth R. Hogstrom, Robert L. Carver, John P. Gibbons, Polad M. Shikhaliev, Kenneth L. Matthews II, Taylor Clarke, Alexander Henderson, and Edison P. Liang. Permanent-magnet energy spectrometer for electron beams from radio-therapy accelerators // Medical Physics. - 2015. - Vol. 42. - No. 9. - P. 5517- 5529.

3. Bødker F Permanent magnets in accelerators can save energy, space and cost // Proc. of IPAC2013. - Shanghai, China, - 2013. P. 3511-3513.

4. Alex Bainbridge, Ben Shepherd, Norbert Collomb, Jim Clarke. Michele Modena The ZEPTO project: Tuneable permanent magnets for the next generation of high energy accelerators // Proc. 25th International Conference on Magnet Technology. Amsterdam, 30th August 2017. TalkID\#448, session Wed-Mo-Or19.

5. Bovda A.M., Guk I.S., Dovbnya A.N., Kononenko S.G., Lyashchenko V.N., Mytsykov A.O. Dipole magnet with a constant field for the accelerator "EPOS" // Problems of Atomic Science and Technology. Series "Nuclear Physics Investigations" 2015. - Vol. 100. - No. 6. - P. 13-17.

6. Beloglasov V.I., Zykov A.I., Zlunitsyn E.S., Kramskoi G.D., Fursov G.L. An electron linac producing beam power up to $15 \mathrm{~kW}$ // Proceedings of the 1996 EPAC. - 1996. - Vol. 1. - P. 798-800.

7. Ayzatsky N.I., Dovbnya A.N. et al. Accelerating system of the technological electron accelerator // Problems of atomic science and technology. Series "Nuclear Physics Investigations". - 2012. - Vol. 80 - No 4. - P. 45-49.

8. Dovbnya A., Guk I., Kononenko S., Tarasenko A. Beam transport system selection on the accelerator LU-10 // Problems of atomic science and technology. Series "Nuclear Physics Investigations". - 2013. - Vol. 88 - No. 6. - P. 57-61.

9. Guk I.S., Kononenko S.G., Lyashchenko V.N., Mytsykov A.O., Choice of parameters of the analyzing magnet for technological electron accelerator LU-10M // Problems of atomic science and technology. Series "Nuclear Physics Investigations". - 2017. Vol. 112. - No. 6. - P. 24-27.

10. Okuda S., Ohashi K., Kobayashi N. Effects of electron beam and y-ray irradiation on the magnetic flux of Nd-Fe-B and Sm-Co permanent magnets // Nucl. Instr. Meth. - 1994. - Vol. B94. - P. 227-230.

11. Luna H.B. et al. Bremsstrahlung radiation effects in rare earth permanent magnets // Nucl. Instr. Meth. - 1989. - Vol. 285. No. 1. - P. 349-354.

12. Alderman J. et al. Measurement of radiation-induced demagnetization of NdFeB permanent magnets // Nucl. Instr. Meth. 2002. - Vol. 481(1-3). - P. 1-3.

13. Dovbnya A. N. et al. Study on radiation resistance of permanent Nd-Fe-B-base magnets under continuous radiation conditions // Problems of Atomic Science and Technology. Series "Nuclear Physics Investigations". - 1999. - Vol. 34. - No. 3. - P. 48-49.

14. Bizen T. et al. Demagnetization of undulator magnets irradiated high energy electrons // Nucl. Instr. Meth. - 2001. - Vol. 467. P. 185-189.

15. Bizen T. et al. High-energy electron irradiation of NdFeB permanent magnets: Dependence of radiation damage on the electron energy // Nucl. Instr. Meth. - 2007. - Vol. 574. - No. 3. - P. 401-406.

16. Qiu R. et al. Radiation damage of $\mathrm{Nd}_{2} \mathrm{Fe}_{14} \mathrm{~B}$ permanent magnets at $2.5 \mathrm{GeV}$ electron accelerator // Nucl. Instr. Meth. - 2008. Vol. 594. - No. 2. - P. 111-118.

17. Gao R.S., et al. Study of $\gamma$-ray irradiation effect on permanent magnets // J. Appl. Phys. - 2008. - Vol. 103. - P. 103-105.

18. Ikeda T., Okuda S. Magnetic flux loss of the permanent magnets used for the wigglers of FELs by the irradiation with highenergy electrons or X-rays // Nucl. Instr. Meth. - 1998. - Vol. 407. - No. 1-3. - P. 439-442.

19. Simos N. et al. An experimental study of radiation-induced demagnetization of insertion device permanent magnets // Proc. EPAC 2008, Genoa, Italy. - 2008. - P. 2112-2114.

20. Vagin P., Bilani O., Schöps A., Tripathi S., Vielitz T., Tischer M. Radiation damage of undulators at PETRA III // Proc. IPAC2014, Dresden, Germany. - 2014. - P. 2019-2021.

21. Rui Qiu, Hee-Seock Lee, Junli Li, Tae-Yeong Koo, T. Bizen \& Qiyong Fan. Demagnetization of Nd-Fe-B permanent magnet at $2.5 \mathrm{GeV}$ electron accelerator // Journal of Nuclear Science and Technology. - 2008. - Vol. 45. - P. 46-49.

22. Bizen T., Asano Y., Maréchal X.-M. SPring-8. Irradiation Experiments and Magnet Protection Plans at SPring-8 // IEEE Editorial Style Manual, IEEE Periodicals, Piscataway, NJ, USA. - Oct. 2014. - P. 34-52.

23. Bizen T. Brief review of the approaches to elucidate the mechanism of the radiation-induced demagnetization // Proc. ERL2011, Tsukuba, Japan. - 2011. - P. 121-126.

24. Bovda V.A. et al. Magnetic field losses in Nd-Fe-B magnets under $10 \mathrm{MeV}$ electron irradiation // Problems of Atomic Science and Technology. Series "Nuclear Physics Investigations". - 2017. - Vol. 109. - No. 3. - P. 90-94.

25. Bovda A.M. et al. Magnetic properties of $\mathrm{Sm}_{2} \mathrm{Co}_{17}$ magnets under $10 \mathrm{MeV}$ electron beam // Problems of Atomic Science and Technology. Series "Nuclear Physics Investigations". - 2017. - Vol. 112. - No. 6. - P. 162-166.

26. Bovda V.A., Bovda A.M., Guk I.S., Dovbnya A.N., Kononenko S.G., Lyashchenko V.N., Mytsykov A.O., Onischenko L.V. Dipole Magnet With A Permanent Magnetic Field For Technological Electron Accelerator // Proceedings of the REPM 2016, Darmstadt, Germany. - 28 August - 1 September 2016. - P. 481-487. 